\title{
OPINION
}

\section{Heavy ion carcinogenesis and human space exploration}

\section{Marco Durante, and Francis A. Cucinotta}

Abstract: Prior to the human exploration of Mars or long duration stays on the Earth's moon, the risk of cancer and other diseases from space radiation must be accurately estimated and mitigated. Space radiation, comprised of energetic protons and heavy nuclei, has been show to produce distinct biological damage compared to radiation on Earth, leading to large uncertainties in the projection of cancer and other health risks, while obscuring evaluation of the effectiveness of possible countermeasures. Here, we describe how research in cancer radiobiology can support human missions to Mars and other planets.

\section{Introduction:}

Space radiation is comprised of high energy protons and high charge (Z) and energy (E) nuclei (HZE), whose ionization patterns in molecules, cells, and tissues, and the resulting initial biological insults are distinct from typical terrestrial radiation. The relationships between the early biological effects of HZE nuclei and the probability of cancer in humans are poorly understood ${ }^{1-3}$, and it is this missing knowledge that leads to large uncertainties in projecting cancer risks (Figure 1), thus representing a major barrier to safe space exploration. Optimizing operational parameters such as the length of space missions, crew selection for age and gender, or applying mitigation measures such as 
radiation shielding or use of biological countermeasures can be used to reduce risk. However, these procedures are clouded by uncertainties, which limit space agencies ability to judge the effectiveness of operational parameters and mitigation measures. Thus, reducing uncertainties by improving our knowledge of cancer radiobiology is the most cost-effective approach for solving the problem of space radiation for the Mars missions and beyond.

Space exploration is a high risk endeavour with the prospects of vehicle or life support system failure always a large concern ${ }^{4,5}$. Acceptable levels of risk must be put into perspective of the value of the mission for humanity and science, the stake-holders for risk acceptance, and the risk-benefit ratio ${ }^{6}$. A goal of less than a $1 \%$ mission failure risk is used in the design of launch, life support, and crew return systems. Radiation risks must be considered in addition to these other mission risks, including the differences in the detriment from a launch failure or a cancer morbidity and death that would be projected to occur several to many years after the mission. The National Aeronautics and Space Administration (NASA) sets risk acceptance levels at 3\% risk of exposure induced death (REID) for radiation carcinogenesis. This level is several times higher than other mission design risk levels, which is appropriate because of the differences in life-loss. Risk levels much higher than 3\% would be difficult to accept because of safety grounds but also because other fatal radiation risks such as heart and digestive diseases ${ }^{7}$ become likely at higher exposure levels, thus making the radiation risk even more problematic. Furthermore, overall uncertainties in space radiation cancer projections are about 5-fold times higher at the $95 \%$ confidence level ${ }^{8,9}$ than the median risk projection and it is not possible, with the current state of knowledge of cancer biology, to judge if risks are 
higher or lower than safety standards. These facts further support that improving the understanding of the biology of cancer risks from space radiation exposure is the primary hurdle for a Mars mission.

Research in this field is also essential for heavy-ion cancer therapy (hadrontherapy), where beams of high-energy carbon ions are used to sterilize solid cancers ${ }^{10-11}$. One major problem related to this treatment is the risk of secondary cancer, especially for pediatric patients ${ }^{12,13}$. Information on heavy ion cancer risk, sought by researchers in space radiation, is also essential to estimate the incidence of secondary malignancies in these patients, and therefore the two research fields share many common topics $^{14}$.

\section{Cancer Risk Estimates From Space Radiation}

Figure 2 provides a physics primer on space radiation types and the differences in energy deposition in biomolecules, cells, and tissues. The differences in physical characteristics of celestial versus terrestrial radiation make the usage of absorbed dose to compare radiation types extremely limited. Nevertheless, epidemiological data, largely from the Atomic bomb survivors in Japan ${ }^{7,15}$ and patients exposed to therapeutic radiation which provides a basis for risk estimation for $\gamma$-ray or X-ray exposure, is presently scaled to heavy ion effects. A key assumption in current methods to project cancer risk is that a radiation cancer mortality rate based on epidemiological studies of the Atomic bomb survivors can be scaled to other populations, dose-rates, and radiation types. Two scaling parameters with large uncertainties are the "radiation quality factor” Q, that estimates the increased effectiveness of HZE nuclei compared to $\gamma$-rays for the same dose, and the 
dose- and dose-rate effectiveness factor (DDREF) that reduces estimates of cancer risk at high dose- and dose-rates when the dose- and dose-rate are low ( $<0.05 \mathrm{~Gy} / \mathrm{hr})$. Risks for extended missions to the moon and the Mars exploration mission exceed 3\% for most Mars mission scenarios with upper 95\% confidence levels near 15\% risk of death ${ }^{9}$. The scaling of mortality rates for space radiation risks to astronauts to the Atomic bomb survivors introduces many uncertainties ${ }^{1,2,8}$ into risk estimates (Figure 1), and there are important questions with regard to the correctness of any scaling approach because of qualitative differences in the biological effects of HZE ions and $\gamma$-rays.

\section{Biological Effectiveness of Energetic Heavy Ions}

\section{Energy deposition}

The initial biophysical events induced by radiation in biomolecules, cells and tissues are key to understanding differences between $\gamma$-rays and the heavy ions in space. Energy deposition by HZE nuclei is highly heterogeneous with a localized contribution along the trajectory of each particle and lateral diffusion of energetic electrons ( $\delta$-rays) many microns from the ions path ${ }^{16,17}$. These particles are therefore densely ionizing, i.e. have a high linear energy transfer (high-LET) along the primary track, however contain a sparsely ionizing (low-LET) component due to the high-energy electrons ejected by ions as they traverse tissue. The dose deposited in a biomolecule is described by microdosimetry concepts such as the mean specific energy and number of events (Figure 2). For both low- and high-LET radiation, mean specific energy of millions of Gray $(G y=J / k g)$ occur for a small DNA segment with heavy ions several times more effective than $\mathrm{X}$-rays ${ }^{17,18}$. Differences between radiation types also occur at the level of 
chromosomal loops or whole chromosomes leading to distinct spatial patterns of DNA lesions for HZE nuclei and X-rays ${ }^{19}$. These differences between radiation types occur due to correlations between damages in nearby sites and the event distribution which can extends out many microns for energetic HZE nuclei.

\section{DNA damage}

Biophysical models show that the energy deposition events by high-LET radiation produce differential DNA lesions, including complex DNA breaks, and that there are qualitative differences between high- and low-LET radiation both in the induction and repair of DNA damage ${ }^{20-23}$. The number of DNA single-strand breaks (SSB) and double-strand breaks (DSB) produced by radiation varies little with radiation type, but for high-LET radiation, a higher fraction of DNA damages are complex, i.e. clusters containing mixtures of two or more of the various types of damages (SSB, DSB, etc.) within a localized region of DNA. Complex damage is uncommon for endogenous damage or low-LET radiation, and has been associated with the increased relative biological effectiveness (RBE) of densely ionizing radiation ${ }^{24,25}$.

The repair of DSB is known to occur through the non-homologous end-joining (NHEJ) and homologous recombination (HR) pathways ${ }^{26-29}$. Foci experiments using immunohistochemistry for anti-bodies of important NHEJ and HR repair proteins have been performed recently with X-rays and HZE nuclei. The histone variant, H2AX, which is a beacon for DSBs, is phosphorylated by members of the phosphoinositide-3-kinaserealted protein kinase (PIKK) family, which includes the catalytic sub-unit of DNA-PK, DNA-PK ${ }_{c s}$, ataxia-telangiectasia mutated (ATM), and the ataxia-telangiectasia and Rad3- 
related (ATR) proteins ${ }^{30}$. The activated form of $\mathrm{H} 2 \mathrm{AX}$, denoted $\gamma-\mathrm{H} 2 \mathrm{AX}$, covers a 2 Mbp region around a DSB ${ }^{31}$. Key differences between X-rays and HZE nuclei are already apparent from these early foci events including a dynamic and larger foci size in the first 2-hrs post-irradiation for HZE nuclei compared X-rays ${ }^{32}$ and the appearance of streaks of foci indicating the path of a particle track, and the slower removal and persistence of foci for HZE nuclei ${ }^{34}$. Other DNA repair proteins ${ }^{35-37}$ such as Artemis ATM, and NBS1 may play a large role in the repair of complex DSBs and are a focus of space radiation research.

Live cell imaging of DNA repair proteins induced with a micro-laser is now widely used to study the kinetics of DNA damage responses ${ }^{38-40}$. Live cell imaging with heavy ions has shown that DNA repair proteins are recruited at sites of heavy ion hits in cell nuclei of fibroblast cells within seconds, and no large scale chromatin movements are associated to the repair activity ${ }^{41}$, yet some movement is observed in the repair protein foci. Image analysis for the dynamics of $\gamma-\mathrm{H} 2 \mathrm{AX}$ and 53pb1 proteins in human epithelial cells fixed following exposure to Fe-ions suggest that DNA lesions are indeed moved to nuclear sub-domains for more efficient repair ${ }^{42}$.

\section{Chromosomal aberrations}

DNA DSBs misrepaired or left unrepaired eventually appear as chromosomal aberrations $^{43}$. Heavy charged particles are very effective at producing chromosomal exchanges with RBE values exceeding 30 in interphase (as visualized using premature chromosome condensation) and 10 at the first post-irradiation mitosis for energetic heavy ions $^{44}$. However, lower values are observed in vivo ${ }^{45-46}$. Besides, cytogenetic studies 
point to a much higher complexity in the chromosomal rearrangements induced by heavy ions compared to sparsely ionizing radiation (Figure 3) - i.e. rearrangements induced by heavy ions involve a higher number of chromosomes and breaks ${ }^{47}$, and include both intra- and inter-chromosomal exchanges ${ }^{48,49}$. Most of these complex rearrangements, however ultimately lead to cell death. In fact, only a few complex exchanges are found in the bone marrow of mice after one week exposure to Fe-ions ${ }^{46}$, and the fraction of aberrant cells in the progeny of human lymphocytes exposed to heavy ions is close to the frequency observed in samples exposed to $\gamma$-rays ${ }^{50}$ (Figure 3, panel E).

Interestingly, chromosomal aberrations can be measured in the astronauts’ blood lymphocytes and can then be used to test dose and risk estimates from current models ${ }^{51}$. In fact, chromosomal aberrations in the blood lymphocytes are considered a validated biomarker of cancer risk ${ }^{51-53}$, and can be used as biodosimeters to estimate equivalent dose in exposed individuals ${ }^{54}$. Biodosimetry studies performed by NASA ${ }^{55-56}$ and in Russian cosmonauts $^{57}$ show that the measured chromosomal rearrangements in crewmembers returning from space flight are consistent with current models, although the biological results are also affected by a large experimental uncertainty at low doses. However, yields of translocations and dicentrics decrease as a function of the time after exposure during the space mission, and it is unclear what the influence of the time since test should be on risk estimates ${ }^{56}$. Besides, for cosmonauts involved in multiple spaceflights, the total yield of aberrations do not seem to be additive ${ }^{51}$, and in experienced cosmonauts with a total of about 2 years in the final yield of the aberrations is close to the measured background before the first flight ${ }^{57}$. The significance of these findings remains to be elucidated. 


\section{Insights into the Molecular Mechanisms of Radiation-Induced Cancer}

The fundamental problem of space radiation risk assessment is the absence of sufficient proof that models of cancer risk sufficiently describe the biology of tumor formation from HZE nuclei, including the overarching problem of extrapolating experimental results to human populations or individuals. There are gaps in knowledge related to space radiation and carcinogenic processes that must be addressed to validate cancer projection models ${ }^{58}$ (Figure 4). Animal studies generally demonstrate that HZE nuclei have a higher carcinogenic effectiveness than low-LET radiation ${ }^{59-62}$, but RBE values are difficult to quantify because of statistical uncertainties, which in many experiments prevents a conclusion on response at low dose and dose-rates. The large number of radiation types and energies in space precludes an extensive study of tumor types in different strains of mice with different ion/dose regimes. In adults the major cancers in exposed cohorts are lung, leukemia, breast, colorectal, and stomach ${ }^{7,15}$, however there are a large number of other cancers and tissues that contribute to the overall risk. There are also differences in background cancers in different countries and ethnic populations making the extrapolation of data from the Japanese A-bomb survivors to a future population of astronauts problematic. Identifying the cell of origin for specific tumor types, and looking for similarities in the mechanisms of tumor initiation, progression and promotion in the mouse or in 3-dimensional human cell culture models of epithelial interacting with stroma cells, may serve as the best model to study differences in radiation qualities and to improve confidence in the model to astronaut risk extrapolation. Humanized mice offer a novel approach to this problem ${ }^{63}$. 
Cat and Mouse Game, or Little Green Men?

Research on carcinogenesis continues to debate a fundamental question on what is cause or effect: DNA damage and mutation leading to genomic instability, or extracellular matrix remodeling and other non-targeted effects? For HZE nuclei such a fundamental question is complicated by what are the consequences of the early events that are already known to be different from X-rays (see Figure 3) and do they lead to an alien architecture on the voyage to malignancy? Tissue effects independent of DNA damage that have been associated with cancer initiation or progression include genomic instability $^{64-68}$, extracellular matrix remodeling ${ }^{69-70}$, persistent inflammation ${ }^{71-72}$, and oxidative damage $\mathrm{e}^{73-74}$. HZE nuclei will likely modify these responses, along with DNA changes, in ways distinct from other radiation types or carcinogens complicating our ability to estimate and mitigate risks.

\section{New Approaches to Cancer Risk Estimates}

Lung cancer makes-up about one-third of the cancers attributable to radiation observed in the Atomic-bomb survivors ${ }^{75}$ and nuclear reactor workers ${ }^{76}$. However, risk estimates are confounded by the use of tobacco by a large fraction of the exposed. Radiation and tobacco are believe to act additively, but the large tobacco risk often precludes identification of the radiation effect with high accuracy ${ }^{75,15}$. A very small number of astronauts have smoked in the past and virtually none at this time. A new experimental model that allows for a novel approach to study individual or combinations of mutations in lung cancer progression has been recently developed ${ }^{77}$ : a 3-dimensional co-culture of 
hTERT-immortalized with cdk4 bypass of p16, bronchial epithelial cells interacting with a stroma layer. Over 30 primary lines have been immortalized to study how specific genetic components influence radiation induced transformation. The results show the extreme inefficiency with which X-rays transform primary cell lines. A recent report of the genetic makeup of lung cancers ${ }^{78}$ in a large collection of tumors shows the complexity of the genetic alterations involved: indeed, many of the genes involved in adenocarcinomas are still unknown. Studies using the 3-D co-culture models with iron nuclei are being carried out as well and will be especially important due to the limitations in animal models of human lung carcinogenesis ${ }^{79}$.

Research on the mechanisms of radiation-induced mammary carcinogenesis have highlighted the importance modifications of tissue regulation in the development of cancer. Transforming growth factor $\beta 1$ (TGF $\beta$ ) is a tumor suppressor during cancer initiations and a promoter during progression ${ }^{80}$. Recent studies of TGF $\beta$ 's role in tumorigenesis highlight how tissues can control a broad range of cellular responses including how knockout of TGF $\beta$ lead to the down-regulation of the DNA damage responses as evidence by almost complete shut-down of $\gamma$-H2AX foci ${ }^{81}$, and induction of an epithelial to mesenchymal transition ${ }^{82}$ after ionizing radiation exposure in a primary human cell culture model.

\section{Countermeasures for Exploration of Mars and beyond}

Even if risk projections and their uncertainties are reduced in the next few years, effective countermeasures to reduce the biological damage produced by radiation remains a long-term goal of space research. Such countermeasures may not be needed for a lunar 
base, but probably for the Mars mission, and definitely for exploring Jupiter or Saturn's moon Titan or the nearby satellites ${ }^{2}$. In all basic radioprotection textbooks, it is stated that there are three means to reduce exposure to ionizing radiation: increasing the distance from the radiation source, reducing the exposure time, and by shielding. Distance plays no role in space, being space radiation is omni-directional. Time in space should be increased rather than decreased according to the plans of exploration and colonization. Shielding remains as a plausible countermeasure, but a prohibitively costly in light of current launch mass capabilities. Furthermore, the present uncertainties in risk projection prevent us from determining the true benefit of shielding. Other strategies can be effective in reducing exposure, or the effects of the irradiation, in space. These strategies include the choice of an appropriate time of flight, administration of drugs or dietary supplements to reduce the radiation effects, and crew selection.

\section{Radioprotective agents}

The search for efficient radioprotectors is a major goal of research in radiation protection and therapy. Both radiation injury and oxygen poisoning occur through the formation of reactive oxygen species, and therefore antioxidants can be efficiently used to prevent the damage ${ }^{83}$.

Phosphorothioates and other aminothiols, which are usually administered shortly before irradiation, are so effective in tissue protection against ionizing radiation that one specific compound (Ethyol, also known as amifostine or WR-2721) is approved in many countries for clinical use during chemotherapy and radiotherapy cancer treatments ${ }^{84}$. Unfortunately, amifostine (WR-2721) and other thiols have significant side effects, 
including nausea, vomiting, vasodilatation, and hypotension ${ }^{85}$. Therefore, it is not possible to use these compounds in space flights for protection against chronic exposure to cosmic radiation. Natural occurring antioxidants are less effective than phosphorothioate agents in protection against high-dose acute radiation burden. However, nutritional antioxidants have a low toxicity, can be used for prolonged time, and they seem to play a key role in the prevention of cancer $^{86-87}$. A diet rich in fruit and vegetables significantly reduced the risk of cancer in the A-bomb survivor cohort ${ }^{88}$. Retinoids and vitamins (A, C, and E) are probably the most well-known and studied natural radioprotectors, but hormones (e.g. melatonin), gluthatione, superoxide dismutase, phytochemicals from plant extracts (including green tea and cruciferous vegetables), and metals (especially selenium, zinc, and copper salts) are also under study as dietary supplements for individuals overexposed to radiation ${ }^{89}$, including astronauts. In addition, there is evidence of a reduced antioxidant capacity during spaceflight, as shown by reduced superoxide dismutase (SOD) and total antioxidant activity in some astronauts returning from missions on the International Space Station ${ }^{90}$.

Understanding the effectiveness of antioxidants in space is complicated by the presence of HZE particles. In principle, antioxidants should provide reduced or no protection against the initial damage from densely ionizing radiation, because the direct effect is more important than free radical-mediated indirect radiation damage at highLET. However, there is an expectation that some benefits should occur for persistent oxidative damage related to inflammation and immune responses ${ }^{69}$. Some recent experiments suggest at least for acute high dose irradiation that an efficient radioprotection by dietary supplements can be achieved even in case of exposure to high- 
LET radiation. Ascorbate reduces the frequency of mutations in human-hamster hybrid cells exposed to high-LET C-ions ${ }^{91}$. Vitamin A strongly reduces the induction of fibroma in rats exposed to swift Fe-ions ${ }^{92}$. Dietary supplementation with Bowan-Birk protease inhibitors ${ }^{93}$, L-selenomethionine or a combination of selected antioxidant agents ${ }^{94}$ could partially or completely prevent the decrease in the total antioxidant status in the plasma of mice exposed to proton or HZE particle radiation, and neoplastic transformation of human thyroid cells in vitro. There is evidence that dietary antioxidants (especially strawberries) can protect the central nervous system from the deleterious effects of high doses of HZE particles ${ }^{95}$. However, because the mechanisms of biological effects are different for low dose-rate compared to acute irradiation, new studies for protracted exposures will be needed to understand the potential benefits of biological countermeasures.

Even if antioxidants can act as radioprotectors, this does not necessarily translate in an advantage for the cancer risk. If antioxidants protect cells by rescuing them from apoptosis, then this may allow the survival of damaged cells, which eventually can initiate tumor progression. Concern on this possibility is sustained by a recent meta-study of the effects antioxidant supplements in the diet of normal subjects ${ }^{96}$. The authors did not find statistically significant evidence that antioxidant supplements have beneficial effects on mortality. On the contrary, they concluded that $\beta$-carotene, vitamin A, and vitamin E seem to increase the risk of death. Concerns not only include rescuing cells that still sustain DNA mutations, but also the altered methylation patterns that can result in genomic instability ${ }^{97}$. An approach to target damaged cells for apoptosis may be advantageous for chronic exposures to galactic cosmic radiation (GCR). Radioprotectors 
tested for acute exposures at high doses should be used with care - rescuing cells may make the problem worst in the long term.

\section{Individual susceptibility}

Studying the mechanisms of genetic sensitivity provides important insights into the understanding of radiation risks to astronauts. Studies of historical data sets such as the atomic-bomb survivors comes with the caveat that sub-sets of the exposed cohorts could have a higher radiation risks and therefore skew the assessments of an average responder in the population upward. Mechanistic studies of DNA repair genes such as ATM, NBS1, and Artemis, and of signal transduction proteins such as TGF- $\beta$, EGFR, and p53 and their impacts on radiation sensitivity improve our understanding of space radiation effects.

Therapeutic misadventures involving ataxia-telangiectasia (AT) patients have dramatically demonstrated the importance of genetic susceptibility to radiation damage in cancer treatment. ATM homozygotes only represent a small fraction of the radiosensitive patients, although they appear to be the most sensitive. ATM heterozygotes, who are also cancer-prone, are suspected to represent a large fraction of the extreme radiosensitive patients $^{98}$. It has been shown that cells heterozygous for ATM mutations are slightly more sensitive to radiation-induced neoplastic transformation than the wild-type ${ }^{99}$. An increased sensitivity of ATM heterozygotes has been also proved in vivo, measuring the induction of cataracts in ATM homozygotes, heterozygotes, and wild-type mice exposed to $0.5-4$ Gy X-rays ${ }^{100}$. Besides ATM, the issue is whether low penetrance genes can determine sensitivity to radiation-induce cancer. A recent study on subjects exposed to 
high radiation doses to treat ringworm of the scalp (tinea capitis) in Israel revealed a strong familiar risk of radiation-induced meningioma ${ }^{101}$, suggesting that radiation carcinogenesis might be an issue for a genetically predisposed subgroup of the general population, rather than a random event ${ }^{102}$.

It is not known if individuals displaying hypersensitivity to ionizing radiation will be hypersensitive to the same level to HZE nuclei as low LET irradiation, or if findings at high dose and dose-rates will hold at low dose-rate and doses. Mice heterozygous for the ATM gene are more sensitive to cataractogenesis than wild-types not only after exposure to X-rays, but also after localized irradiation with high-energy Fe-ions ${ }^{103}$. However these and other studies show that high LET irradiation has a reduced dependence on genetic background compared to low LET irradiation.

A predictive assay able to identify radiation hypersensitive or cancer-prone subjects could be useful in crew selection for long-term space flights. However, this assay is neither scientifically achievable or within society norms in most countries at the present time. Ultimately, for a high risk and high cost endeavor such as a mission to Mars, screening astronauts with increased resistance to space radiation may be sought in order to reduce the costs of the missions.

\section{Shielding}

For terrestrial radiation workers, additional protection against radiation exposure is usually provided through increased shielding. Unfortunately, shielding in space is problematic, especially when GCR is considered. High-energy radiation is very penetrating: a thin or moderate shielding is generally efficient in reducing the equivalent 
dose, but as the thickness increases, shield effectiveness drops (see cancer risk estimates as a function of shielding thickness in Figure 2). This is the result of the production of a large number of secondary particles, including neutrons, caused by nuclear interactions of the GCR with the shield. These particles have generally lower energy, but can have higher quality factors than incident cosmic primary particle.

Contrary to the shielding of photons on Earth, where heavy elements such as lead are preferred, in space shielding effectiveness per unit mass is the highest for hydrogen, and decreases by increasing the target atomic number $A_{T}$. In fact, computer models (such as the HZETRN used by NASA or Geant4 used by ESA), accelerator and flight measurements, clearly show that light, highly hydrogenated materials provide the best protection against space radiation ${ }^{104-108}$. Liquid hydrogen should display the maximum performance as shield material. Hydrogen is not a practical shield material, being a low temperature liquid. So far, it appears that polyethylene $\left(\mathrm{CH}_{2}\right)$ can be a good compromise (Figure 2), and in fact it has been installed in the sleeping quarters of the International Space Station to reduce the dose to the astronauts. Unfortunately, most of the biologically dangerous secondary radiation is produced in tissue by very high energy GCR nuclei even behind hydrogen shielding. With the current technology, active (magnetic) shielding is not yet feasible ${ }^{109-110}$, and passive (bulk) shields are able to reduce the exposure, but probably not to solve the problem ${ }^{105}$.

\section{Summary}

Space radiation, isolation (psychosocial problems), and microgravity-induced physiological changes represent the main health problems for the exploration of the Solar 
system. However, cancer risk caused by exposure to space radiation is now generally considered the main hindrance to interplanetary travel, for the following reasons: large uncertainties are associated with the projected cancer risk estimates; no simple and effective countermeasures are available, and the large uncertainties prevent determining the effectiveness of countermeasures. Research is currently focusing on biological effects of heavy ions, where the highest uncertainty is concentrated, using cellular, tissue, or animal models exposed at large nuclear accelerators. Ground-based experimentation is in fact the key to solve the problem of space radiation, because flight experiments are difficult, expensive, poorly reproducible, the dose rate is too low to get useful data in reasonable time, and experiments in the past have yielded no major findings ${ }^{111-112}$. The NASA-funded Space Radiation Health Program ${ }^{113}$ is built upon the capabilities the NASA Space Radiation Laboratory (NSRL) at the Brookhaven National Laboratory (Upton, NY, USA), and has produced experimental data in the past few years of great relevance for reducing uncertainty on risk assessment. The European Space Agency (ESA) has also recently initiated a ground-based radiobiology program ${ }^{114}$, which will be based at the high-energy synchrotron of the Gesellschaft für Schwerionenforschung (GSI) in Darmstadt (Germany) to foster European research in the field. Translation from basic research to cancer risk assessment is not straightforward, but for the moon base activities cancer risk does not appear to be a showstopper, whereas the uncertainty is still too high for a go/no-go decision on the mission to Mars. Radiation is certainly a major problem for space exploration, but it can be understood and solved. A fruitful NASA-ESA collaboration in accelerator-based research should be fostered in the future years, in order 
to reach a consensus on radiation cancer risk for a Mars mission within the next 10 years or so, and eventually to intensity the research on countermeasures, if urgently needed.

\section{Authors:}

Marco Durante is Director of the Biophysics group at GSI, Planckstraße 1, 64291 Darmstadt, Germany, and is Professor at the University Federico II, Department of Physics, Naples, Italy. Francis A. Cucinotta is Chief Scientist for Radiation Studies at NASA Lyndon B. Johnson Space Center, Houston, TX, 77058, USA. Correspondence to M.D. and F.A.C. E-mails: M.Durante@gsi.de, Francis.A.Cucinotta@nasa.gov.

\section{Acknowledgments}

We thank the NASA Space Radiation Program, the Italian Space Agency (ASI) MoMaCOUNT, and the DoE Low Dose Program for support of the research performed by the authors. Dr. Walter Schimmerling should receive the gratitude of the whole space radiobiology community for initiating and promoting the NASA Space Radiation Health Program. We also acknowledge the commitment of ESA to initiate an European groundbased space radiobiology program (IBER), and the great efforts and enthusiasm of Dr. Oliver Angerer at ESTEC for this project. 


\section{References}

1. Cucinotta, F.A. \& Durante, M. Cancer risk from exposure to galactic cosmic rays: implications for space exploration by human beings. Lancet Oncol. 7, 431-435 (2006).

2. National Council on Radiation Protection and Measurements. Information needed to make radiation protection recommendations for space missions beyond LowEarth Orbit. NCRP Report No. 153, Bethesda, MD (2006).

3. European Space Agency: HUMEX- Study in the Survivability and Adaptation of Humans to Long-Duration Exploratory Missions. ESA-ESTEC SP-1264, Nordwijk, The Netherlands (2003).

4. White, R.J., Averner, M. Humans in space. Nature 409, 1115-1118 (2001).

5. National Academy of Science, Institute of Medicine and National Research Council, Bioastronautics Roadmap: a Risk Reduction Strategy for Human Exploration of Space. Washington D.C. National Academy Press (2005).

6. National Council on Radiation Protection and Measurements: Acceptability of risk from radiation- application to human space flight. NCRP Symposium Proceedings No. 3, Bethesda MD (1997).

7. Preston, D.L., et al. Studies of mortality of atomic bomb survivors. Report 13: Solid cancer and noncancer disease mortality: 1950-1997. Radiat Res 160, 381407 (2003).

8. Cucinotta, F.A. et al. Space radiation cancer risks and uncertainties for Mars missions. Radiat Res 156, 682-688 (2001).

9. Cucinotta, F.A., Kim, M. H. \& Ren., L. Evaluating shielding effectiveness for reducing space radiation cancer risks. Radiat Meas. 41, 1173-1185 (2006).

10. Amaldi, U. \& Kraft, G. Radiotherapy with beams of carbon ions. Rep. Prog. Phys. 68, 1861-1882 (2005).

11. Schulz-Ertner, D. \& Tsujii, H. Particle radiation therapy using proton and heavier ion beams. J. Clin. Oncol. 25, 953-964 (2007).

12. Hall, E.J. Intensity-modulated radiation therapy, protons, and the risk of second cancers. Int. J. Radiat. Oncol. Biol. Phys. 65, 1-7 (2006).

13. Allan, J.M. \& Travis, L.B. Mechanisms of therapy-related carcinogenesis. Nat. Rev. Cancer 5, 943-955 (2005).

14. Durante, M. Heavy ion radiobiology for hadrontherapy and space radiation protection. Radiother. Oncol. 73, S158-S160 (2004).

15. National Academy of Sciences, Committee on Biological Effects of Ionizing Radiation, Health risks from exposure to low levels of ionizing radiation. BEIR VII. National Academy Press, Washington, D.C. (2005).

16. Krämer, M. \& Kraft, G. Calculations of heavy ion track structure. Radiat. Environ. Biophys. 33, 91-109 (1994).

17. Cucinotta, F.A., Nikjoo, H., \& Goodhead, D.T., Model of the radial distribution of energy imparted in nanometer volumes from HZE particles. Radiat. Res. 153, 459-468 (2000). 
18. Goodhead, D.T. Initial events in the cellular effects of ionising radiation: clustered damage in DNA. Int. J. Radiat. Biol. 65, 7-17 (1994).

19. Ponomarev, A. \& Cucinotta, F.A. Chromatin loops are responsible for higher counts of small DNA fragments induced by high-LET radiation, while chromosomal domains do not affect the fragment sizes. Int. J. Radiat. Biol. 82, 293-305 (2006).

20. Prise, K.M. et al. A review of dsb induction data for varying quality radiations. Int J. Radiat. Biol. 74, 173-184 (1998).

21. Sutherland, B.M., Bennett, P.V., Sidorkina, O. \& Laval, J. Clustered DNA damages induced in isolated DNA and in human cells by low doses of ionizing radiation. Proc. Natl. Acad. Sci. USA 97, 103-108 (2000).

22. Rydberg B., et al. Dose-dependent misrejoining of radiation-induced DNA double-strand breaks in human fibroblasts: experimental and theoretical study for high- and low-LET radiation. Radiat. Res. 163, 526-534 (2005).

23. Leatherbarrow, E.L., et al. Induction and quantification of $\gamma-\mathrm{H} 2 \mathrm{AX}$ foci following low and high LET-irradiation. Int. J. Radiat. Biol. 82, 111-118 (2006).

24. Fakir, H., et al. Clusters of DNA double-strand breaks induced by different doses of nitrogen ions for various LETs: experimental measurements and theoretical analyses. Radiat. Res. 166, 917-927 (2006).

25. Hada, M. \& Sutherland, B.M. Spectrum of complex DNA damages depends on the incident radiation. Radiat. Res. 165, 223-230 (2006).

26. Phillips, E.R. \& McKinnnon, P.J. DNA double-strand break repair and development. Oncogene 26, 7799-7808 (2007).

27. Löbrich, M. \& Jeggo, P.A. The impact of a negligent G2/M checkpoint on genomic instability and cancer induction. Nat Rev Cancer. 7, 861-869 (2007).

28. Wyman, C. \& Kanaar, R. DNA double-strand break reapir: all's well that ends well. Annu. Rev. Genet. 40, 363-383 (2006).

29. O’Driscoll, M. \& Jeggo, P.A. The role of double-strand break repair - insights from human genetics. Nat. Rev. Genet. 7, 45-54 (2006).

30. Falck, J, Coates, J., \& Jackson, S.P. Conserved modes of recruitment of ATM, ATR, and DNA-PKcs to sites of DNA damage. Nature 434, 605-611 (2005).

31. Rogakou, E.P. et al. DNA double-strand breaks induce histone H2AX phosphorylation on serine 139. J. Biol. Chem. 273, 5858-5868 (1998).

32. Costes, S.V. et al. Imaging features that discriminate between foci induced by high- and low-LET radiation in human fibroblasts. Radiat. Res. 165, 505-515 (2006).

33. Desai, N. et al. Immunofluorescent detection of DNA double strand breaks induced by high-LET radiation. Radiat. Res. 164, 518-521 (2005).

34. E. Riballo, et al., A pathway of double-strand break rejoining dependent upon ATM, Artemis and proteins locating to $\gamma$-H2AX foci. Mol. Cell. 16, 715-724 (2004).

35. Wang, J. et al. Artemis phosphorylation and function in response to damage. DNA Repair 4, 556-570 (2005).

36. Pluth, J.M., et al. DNA double strand break repair and chromosomal rejoining defects with misrejoining in Nijmegen breakage syndrome cells. DNA Repair 7, 108-118 (2008). 
37. Lukas, C., et al., Mdc1 couples DNA double-strand break repair recognition by Nbs1 with its H2AX-dependent chromatin retention. EMBO J. 23, 2674-2683 (2004).

38. Mari, P.O., et al. Dynamic assembly of end-joining complexes requires interaction between Ku70/80 and XRCC4. Proc. Natl. Acad. Sci. U.S.A. 103, 18597-18602 (2006).

39. Williams, E.S. et al. DNA double-strand breaks are not sufficient to initiate recruitment of TRF2. Nat. Genet. 39, 696 - 698 (2007)

40. Uematsu, N. et al. Autophosphorylation of DNA-PKCS regulates its dynamics at DNA double-strand breaks. J. Cell Biol., 217, 219-229 (2007).

41. Jakob, B. et al. Live cell imaging of heavy-ion-induced radiation responses by beamline microscopy. Radiat. Res. 163, 681-90 (2005).

42. Costes, S.V. et al. Image-based modeling reveals dynamic redistribution of DNA damage into nuclear sub-domains. PLOS Comp. Biol. 3, 1477-1488 (2007).

43. Cornforth, M.N. Perspectives on the formation of radiation-induced exchange aberrations. DNA Repair 5, 1182-1191 (2006).

44. George, K. et al. Biological effectiveness of accelerated particles for the induction of chromosome damage measured in metaphase and interphase human lymphocytes. Radiat. Res. 160, 425-435 (2003).

45. Tucker, J.D., Marples, B., Ramsey, M.J. \& Lutze-Mann, LH. Persistence of chromosome aberrations in mice acutely exposed to ${ }^{56} \mathrm{Fe}^{+26}$ ions. Radiat. Res. 161, 648-655 (2004).

46. Rithidech, K.N, Honikel, L. \& Whorton, E.B. mFISH analysis of chromosomal damage in bone marrow cells collected from CBA/CaJ mice following whole body exposure to ${ }^{56}$ Fe ions. Radiat. Environ. Biophys. 46,137-45 (2007).

47. Durante, M., George, K., Wu, H. \& Cucinotta, F,A., Karyotypes of human lymphocytes exposed to high-energy iron ions. Radiat. Res. 158, 581-590 (2002).

48. Johannes, C. et al. Chromosome intrachanges and interchanges detected by multicolor banding in lymphocytes: searching for clastogen signatures in the human genome. Radiat. Res. 161, 540-548 (2004).

49. Hada, M., Cucinotta, F.A., Gonda, S.R. \& Wu, H. mBAND analysis of chromosomal aberrations in human epithelial cells exposed to low- and high-LET radiation. Radiat. Res. 168, 98-105 (2007).

50. Durante, M., George, K., Cucinotta, F.A. Chromosomes lacking telomeres are present in the progeny of human lymphocytes exposed to heavy ions. Radiat. Res. 165, 51-58 (2006).

51. Durante, M., Biomarkers of space radiation risk. Radiat. Res. 164, 467-473 (2005).

52. Norppa H. et al., Chromosomal aberrations an SCE as biomarkers of cancer risk. Mutat. Res. 600, 37-45 (2006).

53. Boffetta, P. et al. Chromosomal aberrations and cancer risk: results of a cohort study from Central Europe. Am. J. Epidemiol. 165, 36-43 (2007).

54. International Atomic Energy Agency. Cytogenetic Analysis for Radiation Dose Assessment. IAEA Technical report n. 405. IAEA, Vienna (2001).

55. George, K. et al., Chromosome aberrations in the blood lymphocytes of astronauts after space flight. Radiat. Res. 156, 731-738 (2001). 
56. George, K., Willingham, H. \& Cucinotta, F.A., Stability of chromosome aberrations in the blood lymphocytes of astronauts measured after space flight by FISH chromosome painting. Radiat. Res. 164. 474-480 (2005).

57. Durante, M. et al. Chromosome aberration dosimetry in cosmonauts after single or multiple space flights. Cytogenet Genome Res. 103, 40-46 (2003).

58. Hanahan, D. \& Weinberg, R.A. The hallmarks of cancer. Cell 100, 57-70 (2000).

59. Fry, R.J.M. \& Storer, J.B. External radiation carcinogenesis. Adv Radiat Biol 13, 31-91 (1987).

60. Alpen, E.L., Powers-Risius, P., Curtis, S.B. \& DeGuzman, R. Tumorigenic potential of high-Z, high-LET charged-particle radiations. Radiat. Res. 136, 382391 (1993).

61. Dicello, J.F. et al. In vivo mammary tumorigenesis in the Sprague-Dawley rat and microdosimetric correlates. Phys. Med. Biol. 49, 3817-3830 (2004).

62. Ando, K. et al. Tumor induction in mice locally irradiated with carbon ions: a retrospective analysis. J. Radiat. Res. 46, 185-190 (2005).

63. Shultz, L.D., Ishikawa, F. \& Greiner, D.L. Humanized mice in translational biomedical research. Nat. Rev. Immunol. 7, 118-130 (2007).

64. Kadhim, M.A. et al. Transmission of chromosomal instability after plutonium alpha-particle irradiation. Nature 355, 738-740 (1992).

65. Sabatier, L., Dutrillaux, B. \& Martin, M. Chromosomal instability. Nature 357, 548 (1992).

66. Sieber, O.M., Heinimann, K. \& Tomlison, I.P. Genomic instability- the engine of tumorigenesis? Nat. Rev. Cancer 3, 701-708 (2003).

67. Huang, L., Snyder, A.R. \& Morgan, W.F. Radiation-induced genomic instability and its implications for radiation carcinogenesis. Oncogene 22, 5848-5854 (2003).

68. Kadhim, M.A., Hill, M.A. \& Moore, S.R. Genomic instability and the role of radiation quality. Radiat. Prot. Dosim. 122, 221-227 (2006).

69. Park, C.C. et al. Ionizing radiation induces heritable disruption of epithelial cell interactions. Proc. Natl. Acad. Sci. USA 100, 10728-10733 (2003).

70. Barcellos-Hoff, M.H, Park, C. \& Wright, E.G. Radiation and the microenvironment - tumorigenesis and therapy. Nat. Rev. Cancer 5, 867-75 (2005).

71. Kusunoki, T. \& Hayashi, T. Long-lasting alterations of the immune system by ionizing radiation exposure: implications for disease development among atomic bomb survivors. Int. J. Radiat. Biol. 31, 1-14 (2007).

72. Rola, R. et al. High-LET radiation induces inflammation and persistent changes in markers of hippocampal neurogenesis. Radiat. Res. 164, 556-560 (2005).

73. Mothersill, C. \& Seymour, B. Radiation-induced bystander effects--implications for cancer. Nat. Rev. Cancer. 4, 158-164 (2004).

74. Sokolov, M.V., Dickey, J.S., Bonner, W.M., \& Sedelnikova, O.A. gamma-H2AX in bystander cells: not just a radiation-triggered event, a cellular response to stress mediated by intercellular communication. Cell Cycle 6, 2210-2222 (2007).

75. Preston, D.L., et al. Solid cancer incidence in atomic bomb survivors: 1958-1998. Radiat. Res. 168, 1-64 (2007). 
76. Cardis, E., et al. The 15-country collaborative study of cancer risks among radiation workers in the nuclear industry: estimates of radiation-related cancer risks. Radiat. Res. 167, 396-416 (2007).

77. Sato, M., et al. Multiple Oncogenic changes (K-RASv12, p53 knockdown, mutant EGFRs, p16 Bypass, Telomerase) are not sufficient to confer a full malignant phenotype on human bronchial epithelial cells. Cancer Res. 66, 2116-2128 (2006).

78. Weir, B.A., et al. Characterizing the cancer genome in lung adenocarcinoma. Nature 450, 893-898 (2008).

79. National Council on Radiation Protection and Measurements: Extrapolation of Radiation-Induced Cancer Risks from NonHuman Experimental Systems to Humans. NCRP Report No. 150, Bethesda, MD (2005).

80. Berie, B. \& Moses, H.L. Tumour microenvironment: TGFbeta: the molecular Jekyll and Hyde of cancer. Nat. Rev. Cancer 6, 506-520 (2006).

81. Kirshner, J., et al. Inhibition of transforming growth factor-\{beta 1 signaling attenuates ataxia telangiectasia mutated activity in response to genotoxic stress. Cancer Res. 66,10861-10869 (2006).

82. Andarawewa, K.L., et al. Ionizing radiation predisposes nonmalignant human mammary epithelial cells to undergo transforming growth factor beta-induced epithelial to mesenchymal transition. Cancer Res. 67, 8662-8670 (2007).

83. Weiss, J.F. \& Landauer, M.R. Protection against ionizing radiation by antioxidant nutrients and phytochemicals. Toxicology 189, 1-20 (2003).

84. Sasse, A.D., Clark, L.G., Sasse, E.C., \& Clark, O.A. Amifostine reduces side effects and improves complete response rate during radiotherapy: results of a meta-analysis. Int. J. Radiat. Oncol. Biol. Phys. 64, 784-791 (2006).

85. Boccia, R. Improved tolerability of amifostine with rapid infusion and optimal patient preparation. Semin. Oncol. 29, S9-S13 (2002).

86. Halliwell, B. The antioxidant paradox. Lancet 375, 1179-1180 (2000).

87. Bingham, S. \& Riboli, E. Diet and cancer--the European Prospective Investigation into Cancer and Nutrition. Nat. Rev. Cancer 4, 206-215 (2004).

88. Sauvaget, C., Kasagi, F. \& Waldren, C.A. Dietary factors and cancer mortality among atomic-bomb survivors. Mutat. Res. 551, 145-152 (2004).

89. Weiss, J.F. \& Landauer, M.N. Radioprotection by antioxidants. Ann. N.Y. Acad. Sci. 899, 44-60 (2000).

90. Smith, S.M. et al. The nutritional status of astronauts is altered after long-term space flight aboard the International Space Station. J. Nutr. 135, 437-443 (2005).

91. Waldren, C.A., Vannais, D. B. \& Ueno, A.M. A role for long-lived radicals (LLR) in radiation-induced mutation and persistent chromosomal instability: counteraction by ascorbate and RibCys but not DMSO. Mutat. Res. 551, 255-265 (2004).

92. Burns, F.J. et al. Induction and prevention of carcinogenesis in rat skin exposed to space radiation. Radiat. Environ. Biophys. 46, 195-199 (2007).

93. Guan, J. et al. Effects of dietary supplements on the space radiation-induced reduction in total antioxidant status in CBA mice. Radiat. Res. 165, 373-378 (2006). 
94. Kennedy, A.R., Guan, J. \& Ware, J.H. Countermeasures against space radiation induced oxidative stress in mice. Radiat. Environ. Biophys. 46, 201-203 (2007).

95. Rabin, B.M., Joseph, J.A., \& Shukitt-Hale, B. Effects of age and diet on the heavy particle-induced disruption of operant responding produced by a ground-based model for exposure to cosmic rays. Brain Res. 1036, 122-129 (2005).

96. Bjelakovic, G. et al. Mortality in randomized trials of antioxidant supplements for primary and secondary prevention: systematic review and meta-analysis. JAMA. 297, 842-857 (2007).

97. Kovalchuk, O. et al. Methylation changes in muscle and liver tissues of male and female mice exposed to acute and chronic low-dose X-ray-irradiation. Mutat. Res. 548, 75-84 (2004).

98. Thompson, D., et al. Cancer risks and mortality in heterozygous ATM mutations carriers. J. Natl. Cancer Inst. 97, 813-822 (2005).

99. Smilenov, L.B., Brenner, D.J. \& Hall, E.J. Modest increased sensitivity to radiation oncogenesis in ATM heterozygous versus wild-type mammalian cells. Cancer Res. 61, 5710-5713 (2001).

100. Worgul, B.V. et al. Atm heterozygous mice are more sensitive to radiationinduced cataracts than are their wild-type counterparts. Proc. Natl. Acad. Sci. USA. 99, 9836-9839 (2002).

101. Flint-Richter, P., \& Sadetzki, S. Genetic predisposition for the development of radiation-associated meningioma: an epidemiological study. Lancet Oncol. 8, 403-410 (2007).

102. Hall, E.J. Cancer caused by x-rays--a random event? Lancet Oncol. 8, 369-370 (2007).

103. Hall, E.J. et al. The relative biological effectiveness of densely ionizing heavyion radiation for inducing ocular cataracts in wild type versus mice heterozygous for the ATM gene. Radiat. Environ. Biophys. 45, 99-104 (2006).

104. Wilson, J.W. et al. Issues in protection from galactic cosmic rays. Radiat. Environ. Biophys. 34, 217-222 (1995).

105. Simonsen, L.C., Wilson, J.W., Kim, M.H. \& Cucinotta, F.A. Radiation exposure for human Mars exploration. Health Phys. 79, 515-525 (2000).

106. Miller, J. et al. Benchmark studies of the effectiveness of structural and internal materials as radiation shielding for the international space station. Radiat. Res. 159, 381-390 (2003).

107. Durante, M. et al. Cytogenetic effects of high-energy iron ions: dependence on shielding thickness and material. Radiat. Res. 164, 571-576 (2005).

108. Vana, N. et al. Novel shielding materials for space and air travel. Radiat. Prot. Dosim. 120, 405-409 (2006).

109. Parker, E.N. Shielding space travelers. Sci. Am. 294, 40-47 (2006).

110. Spillantini, P. et al. Shielding from cosmic radiation for interplanetary missions: active and passive methods. Radiat. Meas. 42, 14-23 (2003).

111. Kiefer, J. \& Pross, H.D. Space radiation effects and microgravity. Mutat. Res. 430, 299-305 (1999).

112. Durante, M. \& Kronenberg, A. Ground-based research with heavy ions for space radiation protection. Adv. Space Res. 35, 180-184 (2005). 
113. Schimmerling, W., Cucinotta, F.A. \& Wilson, J.W. Radiation risk and human space exploration. Adv. Space Res. 31, 27-34 (2003).

114. Durante, M. et al. Preparatory study of a ground-based space radiobiology program in Europe. Adv. Space Res. 32, 1082-1086 (2007). 
Figure 1. Uncertainties in Projecting Space Radiation Cancer Risks.

Ionizing radiation is a well known carcinogen on Earth. The risks of cancer for X-rays and gamma-rays have been established at doses above $100 \mathrm{mSv}$, albeit there are large uncertainties and ongoing scientific debate about cancer risk at lower doses and at low dose-rates ( $<50 \mathrm{mSv} / \mathrm{h}$ ). The major uncertainties in risk prediction are:

- Radiation quality effects on biological damage related to the qualitative and quantitative differences between space radiation compared to $\mathrm{x}$ rays

- Dependence of risk on dose-rates in space related to the biology of DNA repair, cell regulation, and tissue or organism responses

- Predicting solar particle events (SPE) including temporal, energy spectra, and size predictions

- Extrapolation from experimental data to humans

- Individual radiation-sensitivity factors including genetic, epigenetic, dietary or "healthy worker" effects

The minor uncertainties in risk prediction are:

- Data on galactic cosmic radiation (GCR) environments

- Physics of shielding assessments related to transmission properties of radiation through materials and tissue

- Microgravity effects on biological responses to radiation

- Errors in human data (statistical, dosimetry or recording inaccuracies)

Panel A presents estimates of uncertainties for some of these factors.

Predicting risks to individuals is difficult as there are very few quantitative measures of individual sensitivity. Only a select few individuals enjoy space travel and projecting risks for individuals rather than populations will be of utmost importance for space missions to Mars. The extrapolation from experimental models to humans is perhaps the greatest challenge to cancer risk assessments. The uncertainties are larger for astronauts in space compared to typical exposures on Earth as illustrated in Panel B, which shows the current estimates of cancer risks and 95\% confidence bands for adults of age 40-yr, the typical age of astronauts on space missions for several terresterial exposures and missions on the International Space Station, a lunar colony, and the projections for a Mars mission. 
Figure 2. Space radiation physics and shielding.

Astronauts are exposed to protons and high energy and charge (HZE) ions along with secondary radiation including neutrons and recoil nuclei, produced by nuclear reactions in spacecraft or tissue. The elemental charge of the galactic cosmic radiation (GCR) extends from hydrogen to uranium, however nuclei above iron $(Z=26)$ are very infrequent (panel A). The energy spectrum of the GCR peaks at about $85 \%$ of the speed or light or 1 $\mathrm{GeV} /$ nucleon in energy units, and consequently these particles are so penetrating that shielding can only partially reduce the doses absorbed by the crew. The number of nuclei per unit area is denoted the fluence, $F$. The large ionization power of HZE ions with $Z>2$ makes them the major contributor to the risk, in spite of their lower fluence than protons. The quantity dose (D) represents the amount of energy deposited per unit mass of material (unit is gray: $1 \mathrm{~Gy}=1 \mathrm{~J} / \mathrm{kg}$ ) and is calculated from the fluence by multiplying it by the linear energy transfer (LET), L such that $\mathrm{D}=\mathrm{F} \times \mathrm{L}$. Because the types of radiation in space are very diverse in how they deposit energy, this quantity is a poor descriptor of biological effects. To estimate biological effects it is necessary to scale radiation dose by a quality factor Q(LET), which varies from 1 at low LET to 30 at high LET and then declines at very high values because of what is called over-kill or wasted energy. The quantity $\mathrm{H}=\mathrm{D} \times \mathrm{Q}$ is called dose equivalent and is measured in sievert (Sv). Panel A shows the contribution in fluence, dose, and dose equivalent of different elements in the GCR. In panel B we show what the energy deposition looks like in reality, a stochastic pattern of ionization and excitation points in biomolecules. The LET results from averaging this pattern over macroscopic distances. The panel $\mathrm{C}$ shows how the cancer risk for a mission to Mars varies for increasing amounts of shielding materials after considering the tissue shielding of the human body. Black and red lines represent water and aluminium shield, respectively. Lower curves are median estimates, and upper curves provide the upper 95\% confidence limits. This calculation shows that even very heavy shields will not be able to reduce the risk of a large factor. 
Figure 3. Chromosomal aberrations induced by heavy ions.

Examples of complex-type aberrations induced by energetic Fe-ions in human peripheral blood lymphocytes. Complex rearrangements involve a minimum of 2 chromosomes and 3 breakpoints. A, polycentric chromosomes visualized by mFISH: from left to right, a quadricentric involving both chromosomes 1 , chromosome 9 and chromosome 6; a dicentric of chromosome 1 and 9, with an insertion of chromosome 3; a tricentric involving both chromosomes 6 and chromosome 1 . B, a complex rearrangement in chromosome 5 visualized by mBAND (courtesy of M. Horstmann). One normal chromosome 5 is visible on the bottom, while the other is broken in 3 pieces. Breakpoints can be mapped on chromosome 5 using mBAND. C, multi-aberrant karyotype, including inter-intrachanges in the progeny of lymphocytes exposed to Fe-ions visualized by armspecific mFISH (courtesy of D. Pignalosa). Arm-specific mFISH shows that complexrearrangements can involve both inter- and intra-chromosomal exchanges. D, fraction of complex-type exchanges in lymphocytes following exposure to $\gamma$-rays or Fe-ions. Aberrations were measured by mFISH in prematurely condensed chromosomes, and clearly Fe-ions induce a high fraction of complex-type rearrangements. However, many of these rearrangements are lethal: in fact, the RBE for the induction of aberrant cells (panel E) decreases from interphase (prematurely condensed chromosomes) to first mitosis, and to later cell-cycles (progeny of irradiated cells). This shows that many of the chromosomal rearrangements induced by heavy ions are not transmitted to the progeny of surviving cells, albeit a higher fraction of complex rearrangements is still observed in the survivors. 
Figure 4. Changes en-route to malignancy for space radiation and the hallmarks of cancer. Terrestrial forms of ionizing radiation such as $\mathrm{X}$-rays or gamma-rays induce a large variety of changes to DNA, biomolecules, and the microenvironment of tissues. Researchers have shown that HZE nuclei in space produced both qualitative and quantitative differences in biophysical damage compared to X-rays or gamma-rays. In order to accurately predict cancer risks and design countermeasure for astronaut's, researchers are addressing gaps in knowledge related to how initial and developing biological changes as they relate to the hallmarks of cancer, differ between acute exposures to X-rays or gamma rays where human epidemiology data exists, to those produced by low doses of protons and HZE nuclei. If qualitative differences occur the present approach to risk estimation will have to be abandoned, while if only quantitative differences occur current models can be fine-tuned and uncertainties reduced through improved knowledge and data in experimental models of cancer risk, and confidence in countermeasure effectiveness will be heightened. 
DRAFT Nature Cancer Reviews- Perspective

Fig. 1

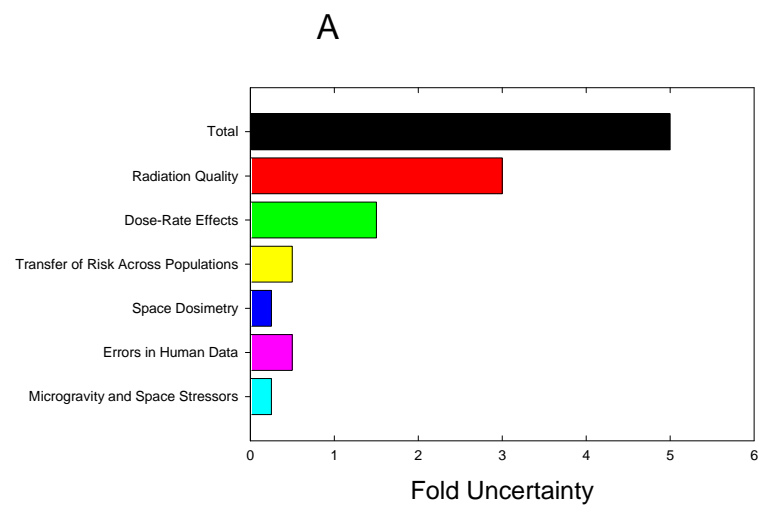

B

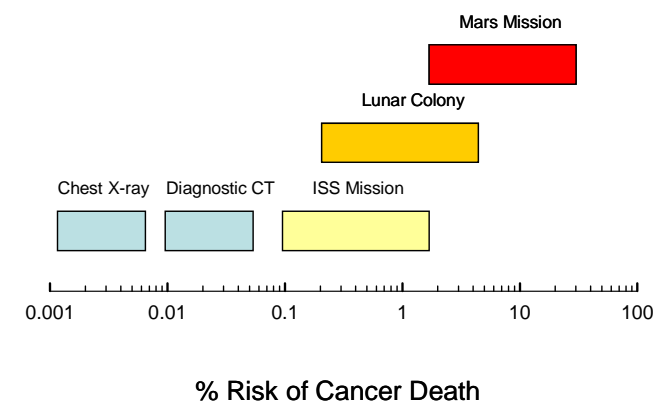


DRAFT Nature Cancer Reviews- Perspective

Fig. 2
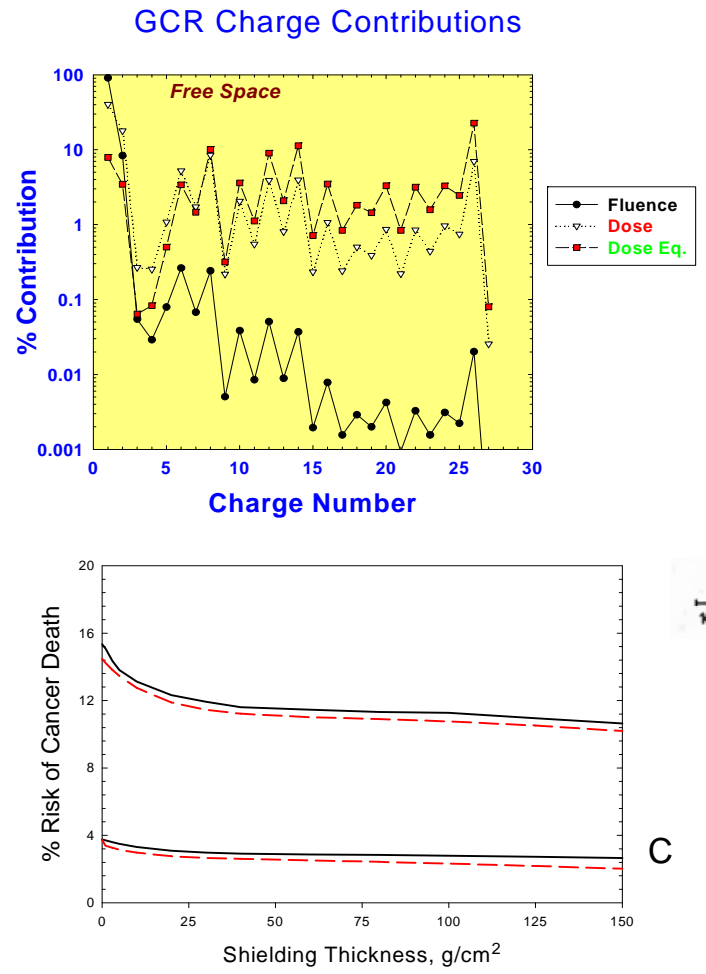

B

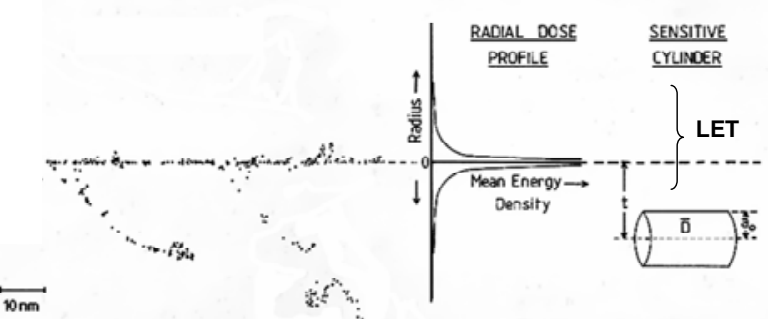


Fig. 3
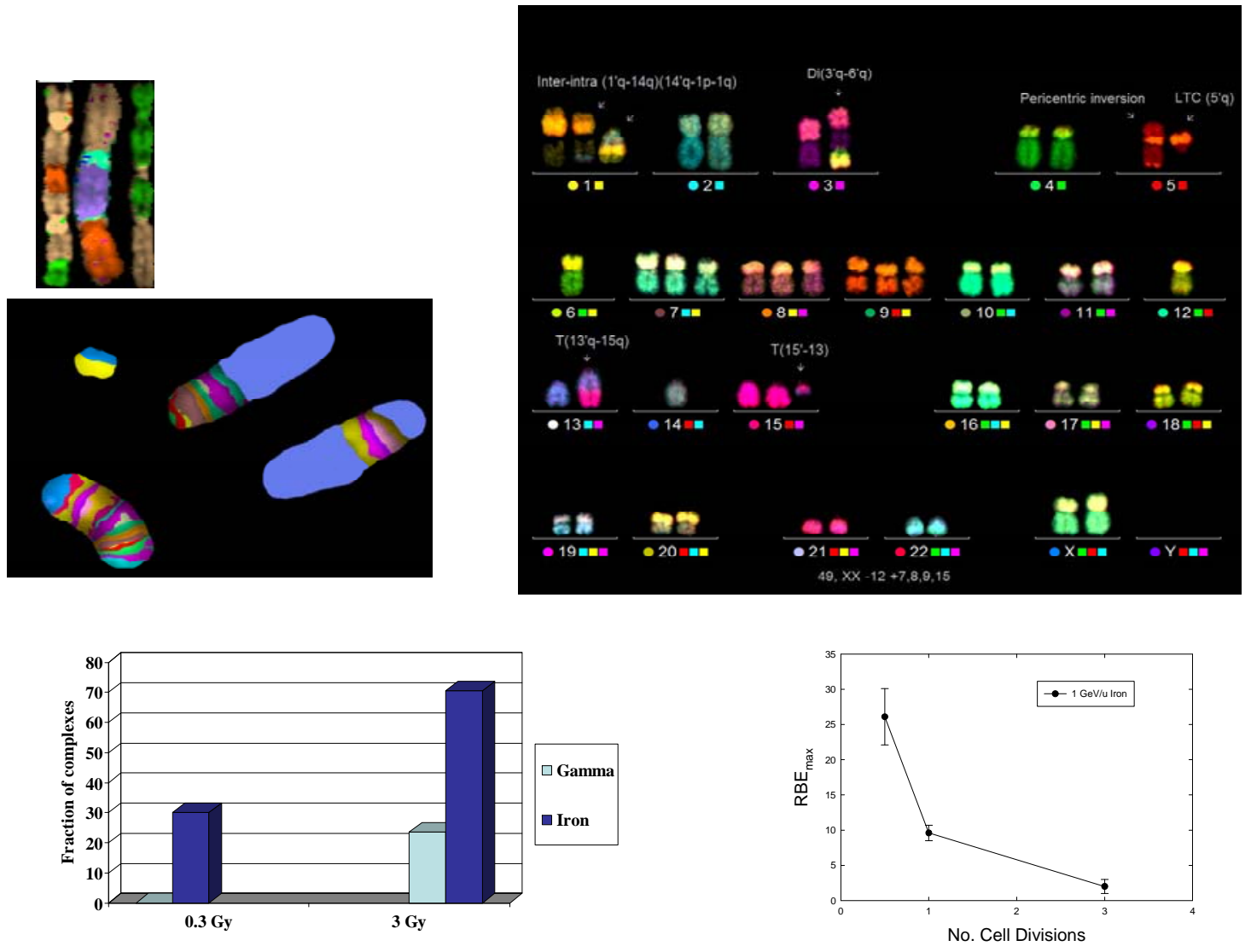
DRAFT Nature Cancer Reviews- Perspective

Fig. 4.

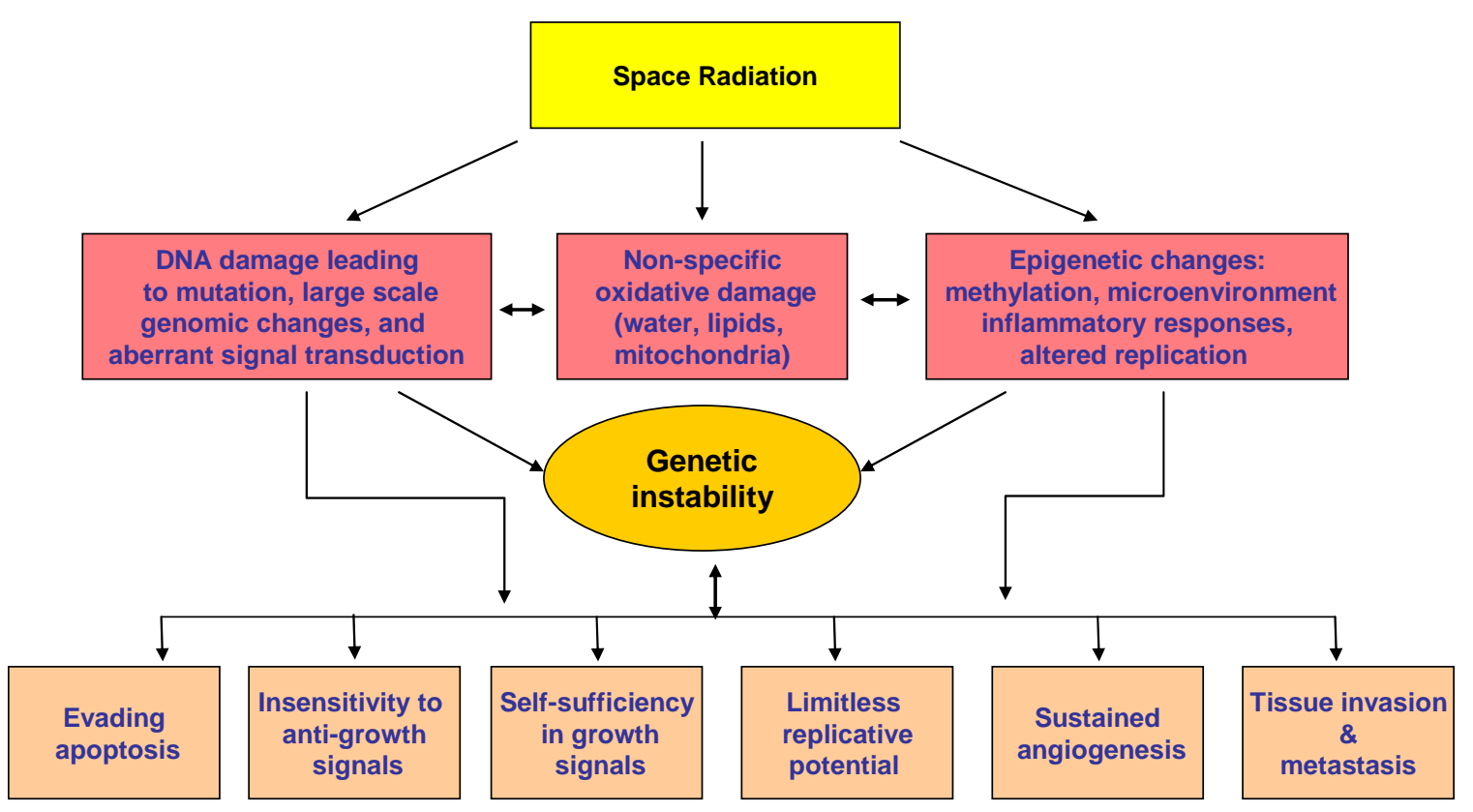

